\title{
Melatonin modulates the effects of diethylstilbestrol (DES) on the anterior pituitary of the female Wistar rat
}

\author{
Weijiang Zhao',2, Zhongfang Shi², Fang Yuan², Guilin Li², Yilin Sun², \\ Yazhuo Zhang', Zhongcheng Wang²
}

${ }^{1}$ Neuroscience Center, Shantou University Medical College, Shantou, Guangdong Province 515041, China

${ }^{2}$ Beijing Neurosurgical Institute, Capital Medical University, Beijing 100050, China

\begin{abstract}
We studied the anti-tumorigenic effect of melatonin in diethylstilbestrol (DES)-treated anterior pituitaries in rats. Twenty-one female Wistar rats were randomly allocated into three groups: vehicle control rats, DES-treated rats, and DEStreated rats co-administrated with melatonin beginning at week 13. At the end of 16 weeks, rats were weighed and decapitated for morphological studies, including an $\mathrm{H}+\mathrm{E}$ staining-based score evaluation in regard to cell proliferation, angiogenesis, immunostaining for VEGF, MMP-9, and AQP-1, and electron microscopy. Compared with vehicle, long-term treatment of DES significantly reduced rat body weight and increased $\mathrm{H}+\mathrm{E}$ score, both of which were counteracted by melatonin. Administration of melatonin also reduced the expression of VEGF and MMP-9, although no changes were detected in AQP1 expression. In rats cotreated with melatonin, the RER loosened and accumulated more secretion granules. We thus concluded that melatonin can modulate the effects of DES on the rat anterior pituitary by downregulating expression of VEGF and MMP-9 and suppressing the release of secretion granules, suggesting a therapeutic potential in estrogen-induced pituitary malfunctions.
\end{abstract}

Key words: melatonin, vascular endothelial growth factor, VEGF, aquaporin-1, AQP-1, MMP-9, ultrastructure

\section{Introduction}

It has been reported that estrogen exposure is one of the factors leading to the development of prolactinoma, a tumor of the pituitary [1]. Experimental estrogen administration can induce prolactinoma in mice and rats, which is characterized by cellular hyperproliferation and angiogenesis, thus providing a means for investigating the mechanisms of pituitary tumorigenesis and effective therapeutic methods against it [2-4].

Melatonin, a neuroendocrine hormone derived from 5-hydroxytryptamine (5-HT) and initially synthesized in the pineal body, is widely distributed in a variety of organs and tissues. Wu et al. [5] demonstrated strong expression of melatonin receptors (MT) in several cerebral nuclei and weak expression of the receptor MT1, through which melatonin might exert its physiological functions and pharmaceutical effects, in both the anterior pituitary and the posterior pituitary glands. In addition, melatonin can protect neurons, enhance immune

Correspondence: F. Yuan and G. Li, Beijing Neurosurgical Institute, Capital Medical University, Beijing 100050, China; e-mail: florayuan@vip.sina.com, liguilin40@hotmail.com function, delay senescence, and regulate cell division $[6,7]$. The antiproliferative effects of melatonin were also observed in multiple carcinoma cell lines of human and mouse, as well as in diethylstilbestrol (DES)induced prolactinoma [8]. Recent investigations show that melatonin suppresses the expression of estrogen receptor (ER), inhibits ER binding to the estrogen response element (ERE), reduces mutations in the estrogen enhancer, and induces apoptosis of prolactinoma cells, leading to reductions in tumor volume [911]. However, the molecular mechanisms of melatonin treatment remain unclear.

Angiogenesis is a typical characteristic of pituitary tumors and is a pivotal event in tumor invasion $[12,13]$. The roles of VEGF, MMP-1, and AQP-1 have been established in angiogenesis, suggesting roles in tumor behavior [14-16]. Lissoni et al. [17] evaluated the effect of melatonin on senior cancer patients, discovering that control of tumor growth by melatonin was related to reduced serum VEGF concentrations, which suggested that melatonin functions as a natural anti-angiogenesis agent to inhibit tumor growth. However, we are not aware of any prior reports on the effect of DES on angiogenesis in pituitary tumors. 
In the present study, we investigated the effect of melatonin on DES-induced expression of VEGF, MMP-9, and AQP-1 and its possible effect on the ultrastructure of the anterior pituitary. Compared with rats without melatonin treatment, rats concurrently receiving melatonin and DES demonstrated significantly reduced expression of VEGF and MMQ-9, accompanied by diminished ultrastructural deterioration of the pituitary tissue. Our findings suggest beneficial effects of melatonin in estrogen-related pituitary malfunction.

\section{Materials and methods}

Animals and materials. Animal manipulation was approved by the Animal Use Committee of Beijing Neurosurgical Institute. A total of 21 female Wistar rats, 3 weeks old, and weighing 70-80 g were used. All animals were housed with free access to tap water and standard pellet food. They were kept at a controlled temperature $\left(24 \pm 1^{\circ} \mathrm{C}\right)$ and humidity $(55 \pm 5 \%)$, and a 12-hour day-night cycle (10 a.m.-10 p.m.) was maintained. Rats were randomly divided into 3 groups of 7 , with each group receiving a different treatment regimen: the vehicle control group, in which rats were intraperitoneally administrated with sunflower seed oil $(1 \mathrm{~mL} / \mathrm{kg}$, twice a week) for 16 weeks; the DES group, which received DES $(5 \mathrm{mg} / \mathrm{kg}$, twice a week) for 16 weeks; and the DES + melatonin group, in which DES was administered for 16 weeks at the same dose as in the DES group, with melatonin ( $1 \mathrm{mg} /$ day) co-administered at 5 p.m. each day from weeks 13 to 16 . Rats in both vehicle and DES groups received the same dose of vehicle in place of melatonin, while the DES + melatonin group received melatonin.

DES and melatonin were purchased from Sigma Chemical Co. (St Louis, MO, USA). Anti-VEGF antibodies were purchased from Boster Biotech (Wuhan, China). MMP-9 and prolactin (PRL) antibodies were purchased from Dako (Denmark). AQP-1 antibody was purchased from Chemicon (Temecula, CA, USA). The DES injection was prepared by dissolving DES powder in sunflower seed oil to obtain a final concentration of $5 \mathrm{mg} / \mathrm{mL}$.

Morphological score of anterior pituitary gland. After 16 weeks, rodents were anesthetized with chloroaldehyde, $10 \%$ hydration $(300 \mathrm{mg} / \mathrm{kg})$ and perfused intracardially with a $40 \mathrm{~g} / \mathrm{L}$ solution of paraformaldehyde in PBS $(0.01 \mathrm{M}, \mathrm{pH} 7.4)$. Tissues were then embedded in paraffin, sectioned at $4 \mu \mathrm{m}$ for $\mathrm{H}+\mathrm{E}$ staining, and observed immunohistochemically. An evaluation score system based on $\mathrm{H}+\mathrm{E}$ staining was employed to compare the changes in the anterior pituitary gland between each group.

Immunohistochemistry. After deparaffinization, sections were rehydrated through a graded series of ethanol to phosphate buffer. For antigen retrieval, the sections were incubated in $3 \% \mathrm{H}_{2} \mathrm{O}_{2}$ to clear endogenous peroxidase and were then saturated with $10 \%$ normal goat serum for 10 minutes. Afterwards, sections were individually incubated with anti-VEGF, -MMP-9, or -AQP-1 antibody (1:50 dilution) at $4^{\circ} \mathrm{C}$ overnight. The antigen-antibody complexes were visualized using the avidin-biotin-peroxidase complex (ABC) method. Counterstaining was done with Meyer's hematoxylin. Table 1 depicts the $\mathrm{H}+\mathrm{E}$ scoring system used.

Transmission electron microscopy. One rat in each group was randomly selected for transmission electron microscopic investigation. For electron microscopy, pituitary tissues were fixed in $2 \%$ glutaraldehyde in $0.1 \mathrm{M}$ PBS (pH 7.4) and then subjected to postfixation in $2 \%$ osmium tetroxide. Tissues were embedded in Araldite, and the ultra-thin sections were examined under a Philips EM208 electron microscope.
Table 1. Score based on $\mathrm{H}+\mathrm{E}$ staining.

\begin{tabular}{|c|l|}
\hline Score & \multicolumn{1}{|c|}{ Description } \\
\hline 1 & No apparent morphological changes \\
\hline 2 & $\begin{array}{l}\text { Structures demonstrating gland cavity narrowing and } \\
\text { cell proliferation occupy }<50 \% \text { of the section stained }\end{array}$ \\
\hline 3 & $\begin{array}{l}\text { Structures demonstrating gland cavity narrowing and } \\
\text { cell proliferation occupy } \geq 50 \% \text { of the section stained }\end{array}$ \\
\hline 4 & $\begin{array}{l}\text { Structures demonstrating gland cavily disappearance } \\
\text { and angiogenesis occupy }<50 \% \text { of the section stained }\end{array}$ \\
\hline 5 & $\begin{array}{l}\text { Structures demonstrating gland cavity disappearance } \\
\text { and angiogenesis occupy } \geq 50 \% \text { of the section stained }\end{array}$ \\
\hline 6 & $\begin{array}{l}\text { Complete tumor-like changes, with no apparent } \\
\text { cavity-like structure }\end{array}$ \\
\hline
\end{tabular}

Ethical issues. The study was monitored by the Bioethical Committee of Beijing Tiantan Hospital, Capital Medical University, China. The number of the license for animal use is SYXK (Beijing) 2008-005.

Statistical analysis. Results were expressed as mean \pm standard deviation (SD), and one-way ANOVA analysis was used to compare differences in body weight and $\mathrm{H}+\mathrm{E}$ score, with post-hoc testing by Bonferroni test. Frequency distribution was applied to test the normal distribution of data in each group using Skewness method. P-values of less than 0.05 were considered significant.

\section{Results}

\section{Physical and histological changes induced by DES and melatonin}

Fur loss and weight loss are the initial superficial effects of intraperitoneal injection of DES in rats. Compared with continuously DES-treated rats, melatonin treatment appeared to reduce fur loss. The body weights of rats in the vehicle control group, the DES group, and the DES + melatonin group were $282.9 \pm 35.6 \mathrm{~g}, 239.9 \pm 20.7 \mathrm{~g}$, and $248.7 \pm 17.4 \mathrm{~g}$, respectively. The frequency of values in each group was distributed in a normal mode. Compared with controls, continuous treatment of DES without melatonin significantly reduced body weight, which was mitigated by coadministration of melatonin, although no significance was observed.

In six out of seven vehicle-treated rats, pituitary tissues showed no apparent abnormalities or gland cavity narrowing, and the pituitary cells were arranged normally. One rat showed self-developed pituitary hyperproliferation. By contrast, pituitary tissues in rats receiving continuous administration of DES showed a disappearance of the gland cavity, with subsequent occupation by proliferated cells. Co-administration of melatonin reduced structural abnormalities, with increased gland cavity volume compared with the DES 
V

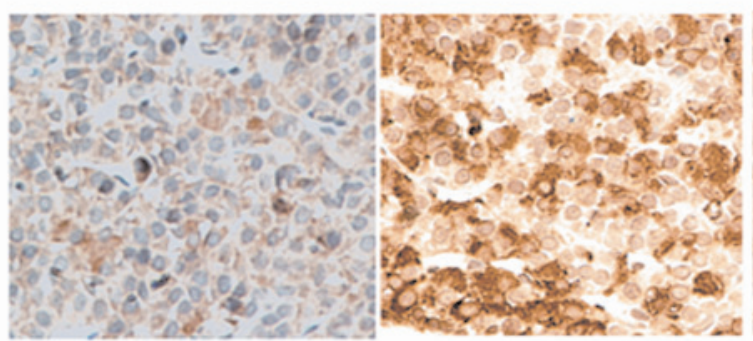

PRL

VEGF
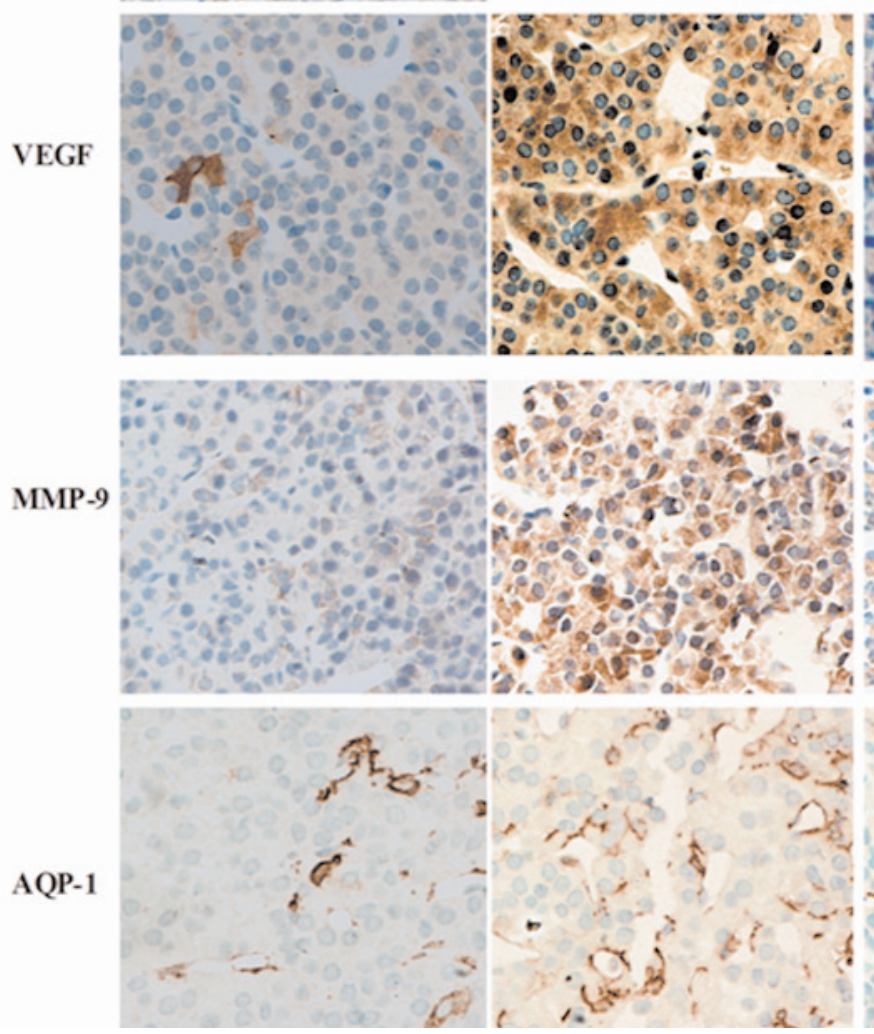

DES+Melatonin

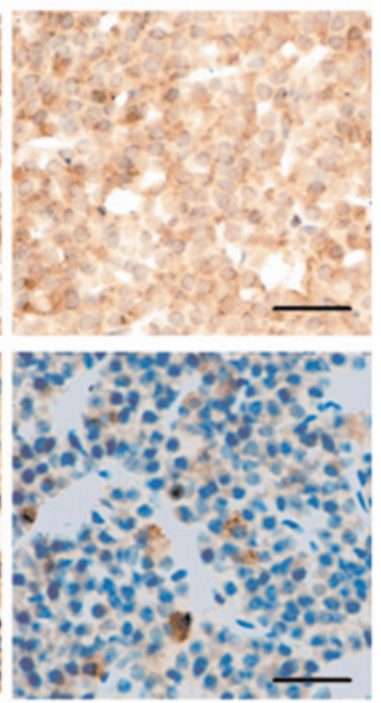

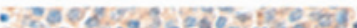

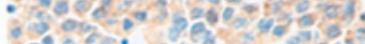

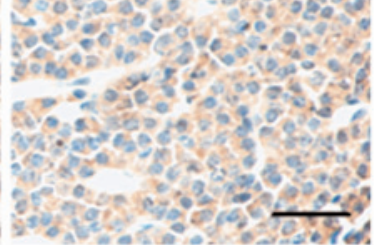

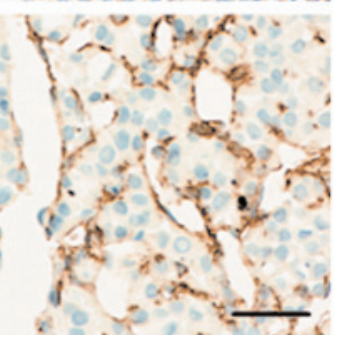

Fig. 1. Immunohistochemical staining of female Wistar rat anterior pituitary gland for expression of PRL, VEGF, MMP-9, and AQP-1. V-vehicle control rats, DES-diehylstilbestrol treated rats, DES+Mdiehylstilbestrol treated rats coadministrated with melatonin. scale bars $=30 \mu \mathrm{m}$.

group, possibly attributed to the sharp withdrawal of proliferated cells.

The $\mathrm{H}+\mathrm{E}$ staining-based evaluation scores in the vehicle, DES, and DES + melatonin groups were $1.42 \pm 1.13,4.57 \pm 0.98$, and $2.00 \pm 0.63$, respectively, and frequency distribution of values in each group were in agreement with normal distribution. Longterm treatment of DES significantly increased the $\mathrm{H}+\mathrm{E}$ score, which was reduced by subcutaneous injection of melatonin ( $<<0.01 v s$. DES group). In the sole pituitary with spontaneous tumor-like changes, the tumor-like cells were closely packed with similar cytoplasmic and nuclear volumes, suggesting that they were from the same origin.

\section{Effects of melatonin on VEGF, MMP-9, and $A Q P-1$}

In the vehicle-treated pituitary tissues, VEGF was selectively expressed in pituitary cells surrounding blood vessels. By contrast, administration of DES led to extensive expression of VEGF in the cytoplasm of pituitary cells, accompanied by angiogenesis, which was reduced by melatonin at a dose of $1 \mathrm{mg}$ /day for 4 weeks (Fig. 1).

No apparent expression of MMP-9 was observed in vehicle-treated rats, whereas administration of DES produced diffuse expression of MMP-9. Co-administration of melatonin abolished MMP-9 expression in the pituitary (Fig. 1). At week 16, expression of AQP1 was increased in the endothelial cells of the pituitary glands of rats receiving DES, which could not be altered by melatonin administration (Fig. 1).

\section{Changes in ultrastructure}

We previously reported in the DES-treated pituitary, electron microscopic investigation revealed welldeveloped rough endoplasmic reticulum (RER) and faulty exocytosis in some cells, indicating the devel- 


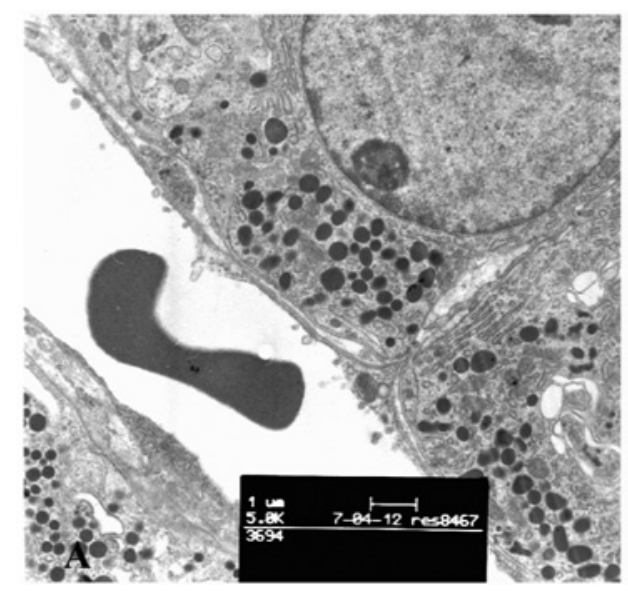

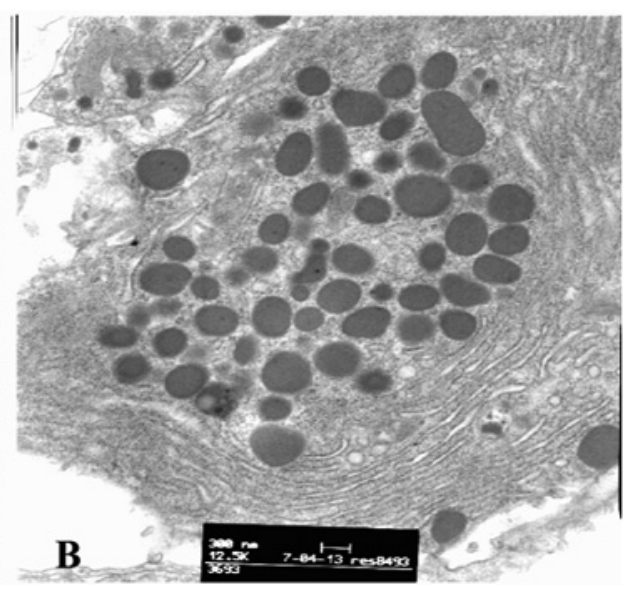

Fig. 2. Electron microscopy on pituitary tissues. Accumulated secretion granules for extrusion into the blood vessels were observed in the DES treated rat pituitary gland (A), whereas in the DES treated rat pituitary co-administrated with melatonin, most of these granules were seen in the loosened structure of the rough endoplasmic reticulum (B). opment of prolactinoma [16]. Here, we also observed the accumulation of secretion granules approaching the blood vessels in the DES treated rat pituitary gland (Fig. 2A). In pituitary tissues of rats co-treated with melatonin, many premature, abnormally enlarged, medium-density hormone containing granules were observed in loosened endoplasmic reticulum, with an average diameter of $300 \mathrm{~nm}$ (Fig. 2B), suggesting melatonin may function by inhibiting the granule extrusion.

\section{Discussion}

Prolactinoma is a multi-factorial disease. Although dopamine agonists, including bromocriptine, quinagolide, and lisuride, have proved to be effective in most patients suffering from prolactinoma, there are still many patients who show no response to these drugs or who develop resistance to them [18]. In this investigation, rats were subjected to DES administration with or without concurrent melatonin treatment, after which immunohistochemistry and electron microscopy were undertaken to investigate the effects of melatonin on the expression of VEGF, MMP-9, and AQP-1, as well as its effect on the ultrastructure of pituitary tissues. Our results demonstrate the ability of melatonin to counteract DES-induced upregulation of VEGF and MMP-9 expression and its ability to improve the ultrastructure of DES-treated pituitary tissue.

Although investigations have demonstrated that the pineal body influences PRL release, little is known about the effects of the pineal body on hyperprolactinemic diseases, and pineal body activity is usually excluded from clinical investigations of pituitary tissues. Lissnoi et al. [19] investigated the effects of melatonin injection on serum PRL in 19 patients with hyperprolactinemia. They found that intramuscular injection of melatonin led to the reduction of serum PRL in 3 out of 8 patients with congenital hyperprolacthemia. Subsequent investigation [17] showed that the serum mela- tonin level in these patients was significantly lower than in normal controls. Additionally, patients with acromegaly and pituitary tumors showed no response in terms of serum growth hormone or prolactin to acute administration of melatonin. Thus, the effects of longterm melatonin treatment need further elucidation.

Xu et al. $[9,20]$ used controllable pumps containing estradiol to induce the development of prolactinoma in rats, which received melatonin treatment from 7 days before implantation to 90 days after implantation. The results demonstrated that melatonin could effectively abolish the growth of prolactinoma by inhibiting PRL and ER expression and could partially prevent the binding of ER to ERE.

Melatonin, at an appropriate dose, can also reduce mutations in the PRL gene enhancer, preventing overexpression of PRL and resulting in the decreased growth of prolactinomas [10]. Further investigations demonstrated that melatonin can increase the activity of caspase-3, increasing the expression of Bcl-2 and facilitating decreases in mitochondrial membrane potential, which promote apoptosis and reduce tumor mass [11,21]. Notably, melatonin treatment was initiated before DES induction in previous studies. In contrast to this preventive mode of treatment, we administered melatonin after 12 weeks of DES pre-treatment.

Co-administration of melatonin with DES could promote the re-emergence of the adenoid cavity, especially in the areas approaching the margin of the anterior pituitary gland. This may be caused by the rapid retreat of proliferative cells, allowing greater blood flow and thus leaving a larger cavity. It can be speculated that further increases in blood flow might rupture the vessels. Therefore, an effective dose of melatonin will need to be determined to optimize vascular stability during melatonin treatment.

Electron microscopy is a sensitive and reliable method for observing the earliest ultrastructural changes related to the development and treatment of a pituitary tumor. Using immune electron microscopy, 
Osamura et al. [22] found that PRL was located in the well-developed, double-layered, concentric rough endoplasmic reticulum. Bromocriptine caused the cytoplasm to shrink and unsecreted PRL granules to aggregate, with the abrogation of exocytosis. Eljarmak et al. [23] found that continuous bromocriptine administration reduced the volumes of the cell, the rough endoplasmic reticulum, and the Golgi complex. The ultrastructural improvements conferred by melatonin provided strong evidence for its efficacy as a therapeutic. Melatonin has been shown to reduce tumor size and serum PRL concentration without the misplaced exocytosis or disruption of RER.

In the present study, administration of melatonin for 4 weeks caused the accumulation of large PRL secretory granules in loosened RER, suggesting melatonin may inhibit the exocytosis of PRL-containing granules and reduce the expression of PRL through negative feedback. The structural improvements to the concentric RER support this.

The anti-cancer activity of melatonin, primarily a product of the pineal body and a natural tumor inhibitor, has been related to its immune-regulatory, anti-proliferation, and anti-oxidant effects. Angiogenesis is a key step in the development and metastasis of most tumors, including pituitary tumors. However, compelling data concerning melatonin in angiogenesis are not yet available. Soybir et al. [24] found that melatonin could function in angiogenesis and wound repair, which was related to the regulatory effects of melatonin on monocytes, cytokines, and fibroblastoma cells. Cui et al. [25] found that melatonin could inhibit the growth of cultured umbilical vein endothelial cells, inducing apoptosis by regulating the cell size, which involved $\mathrm{p} 53$ and $\mathrm{Bax} / \mathrm{Bcl}$ expression changes. EGF and VEGF are involved in angiogenesis, stimulating cellular proliferation, promoting vascularization, and sustaining the integrity of vessels, meaning these proteins play an important role in tumor metastasis [26-28]. The present study demonstrated DESinduced upregulation of VEGF and MMP-9, confirming a role for estrogen in angiogenesis in the rat anterior pituitary. Melatonin has been reported to downregulate MMP-9 expression and activity in several pathological states in rodents, such as reperfusioninduced hemorrhage following transient focal cerebral ischemia and spinal cord injury [29-31]. The inhibitory effects of melatonin may be associated with reduced expression of TNF- $\alpha$ and elevated expression of tissue inhibitor of metalloproteinase (TIMP)-1 [30,32].

Melatonin reduced the expression of both VEGF and MMP-9, accompanied by an improvement in $\mathrm{H}+\mathrm{E}$ score, suggesting these molecules are targets of melatonin in the treatment of estrogen-induced pituitary malfunction. Further study is needed to determine why AQP-1 was upregulated by DES and not suppressed by melatonin.

\section{References}

[ 1] Gooren LJ, Assies J, Asscheman H, de Slegte R, van Kessel H. Estrogen-induced prolactinoma in a man. J Clin Endocrinol Metab. 1988;66:444-446.

[2] Kaplan SE, De Nicola AF. Protein and RNA synthesis in pituitary tumors from F344 rats given implants of estrogen. J Natl Cancer Inst. 1976;56:37-42.

[3] Katznelson L. Hypogonadism in patients with acromegaly: data from the multi-center acromegaly registry pilot study. Clin Endocrinol. 2001; 54:183-188.

[4] Walker BE. Tumors in female offspring of control and diethylstilbestrol-exposed mice fed high-fat diets. $J$ natl Cancer Inst. 1990;82:50-54.

[ 5] Wu YH, Zhou JN, Balesar R. Distribution of MT1 melatonin receptor immunoreactivity in the human hypothalamus and pituitary gland: colocalization of MT1 with vasopressin, oxytocin, and corticotropin-releasing hormone. J Comp Neurol. 2006;499(6):897-910.

[6] Alvira D, Tajes M, Verdaguer E. Inhibition of the cdk5/p25 fragment formation may explain the antiapoptotic effects of melatonin in an experimental model of Parkinson's disease. $J$ Pineal Res. 2006;40(3):251-258.

[ 7] Macchi MM, Bruce JN. Human pinel physiology and functional significance of melatonin. Front Neuroendocrinol. 2004;25:177-195.

[ 8] Karasek M, Pawlikowski M. Antiproliferative effects of melatonin and CGP 52608. Biol Signals Recept. 1999;8:75-78.

[ 9] Gao L, Xu RK, Pang CS. Inhibitory effect of melatonin on the development of pituitary prolactin-producing tumors induced by 17-beta-estradiol. Zhongguo Yi Хие Ке Хие Yиап Хие Вао. 2001;23:49-53.

[10] Gao L, Yang QH, Xu RK. Melatonin inhibits the proliferation of pituitary prolactin-secreting tumor by suppressing the enhancer elements mutation of PRL gene in the rat. Sheng $\mathrm{Li}$ Хие Bao. 2005;57:319-327.

[11] Yang QH, Xu JN, Xu RK. Antiproliferative effects of melatonin on the growth of rat pituitary prolactin-secreting tumor cells in vitro. J Pineal Res. 2007;42:172-179.

[12] Turner HE, Nagy Z, Gatter KC, Esiri MM, Harris AL, Wass JA. Angiogenesis in pituitary adenomas and the normal pituitary gland. J Clin Endocrinol Metab. 2000;85:1159-1162.

[13] Turner HE, Nagy Z, Gatter KC, Esiri MM, Harris AL, Wass JA. Angiogenesis in pituitary adenomas - relationship to endocrine function, treatment and outcome. J Endocrinol. 2000;165:475-481.

[14] Horiguchi H, Jin L, Ruebel KH, Scheithauer BW, Lloyd RV. Regulation of VEGF-A, VEGFR-I, thrombospondin-1, -2, and -3 expression in a human pituitary cell line (HP75) by TGFbeta1, bFGF, and EGF. Endocrine. 2004;24:141-146.

[15] Lloyd RV, Scheithauer BW, Kuroki T. Vascular Endothelial Growth Factor (VEGF) Expression in Human Pituitary Adenomas and Carcinomas. Endocr Pathol. 1999;10:229-235.

[16] Zhao W, Shen H, Yuan F, Li G, Sun Y, Shi Z, Zhang Y, Wang Z. Induction stage-dependent expression of vascular endothelial growth factor and aquaporin-1 in diethylstilbestrol-treated rat pituitary. Eur J Histochem. 2009;53:53-60.

[17] Lissoni P, Rovelli F, Malugani F. Anti-angiogenetic activity of melatonin in advanced cancer patients. Neuroendocr Let. 2001;22:45-47.

[18] Giustina A. criteria for cure of acromegaly: a consensus statement. J Clin Endocrinal Metab. 2000;85:526-529.

[19] Lissoni P, Mainini E, Rovelli F. A clinical study of the pineal hormone melatonin in patients with growth hormone or prolactin secreting pituitary tumours. Eur J Med. 1992;1:407-410.

[20] Xu RK, Guo CH, Huang MY. Induction of rat prolactinoma by $17-\beta$-estradiol and its relation to expression of c-myc oncogene. Dev Reprod Biol. 1995;4:64-69. 
[21] Yang QH, Xu JN, Xu RK. Inhibitory effects of melatonin on the growth of pituitary prolactin-secreting tumor in rats. $J$ Pineal Res. 2006;40:230-235.

[22] Osamura RY, Watanabe K. Ultrastructural localization of prolactin in estrogen-induced prolactinoma of the rat pituitary. Experimental models for the human prolactinomas and the effects of bromocriptine. Acta Pathol Jpn. 1986;36:1131-1137.

[23] Eljarmak D, Lis M, Cantin M. Effects of chronic bromocriptine treatment of an estrone-induced, prolactin-secreting rat pituitary adenoma. Horm Res. 1985;21(3):160-167.

[24] Soybir G, Topuzlu C, Odabas O. The effects of melatonin on angiogenesis and wound healing. Surg Today. 2004;33:896-901.

[25] Cui P, Luo Z, Zhang H. Effect and mechanism of melatonin's action on the proliferation of human umbilical vein endothelial cells. J Pineal Res. 2006;41:358-362.

[26] DeLisser KH, Christofidou-Solomidou M, Strierter RM. Involvement of endothelial PECAM/ CD31 in angiogenesis. Am J Pathol. 1997;151(3):671-674.

[27] LeRiche VK, Asa SL, Ezzat S. Epidermal growth factor and its receptor (EGF-R) in human pituitary adenomas: EGF-R correlates with tumor aggressiveness. J Clin Endocrinol Metab. 1996;81:656-662.
[28] Loyhrer P, Gloddek J, Hopfner U. Vascular endothelial growth factor production and regulation in rat and human pituitary tumor cells in vitro. Neuroendocrinol. 2001;74:95-105.

[29] Esposito E, Genovese T, Caminiti R, Bramanti P, Meli R, Cuzzocrea $\mathrm{S}$. Melatonin regulates matrix metalloproteinases after traumatic experimental spinal cord injury. J Pineal Res. 2008;45:149-156.

[30] Esposito E, Mazzon E, Riccardi L, Caminiti R, Meli R, Cuzzocrea S. Matrix metalloproteinase- 9 and metalloproteinase-2 activity and expression is reduced by melatonin during experimental colitis. J Pineal Res. 2008;45:166-173.

[31] Hung YC, Chen TY, Lee EJ, Chen WL, Huang SY, Lee WT, Lee MY, Chen HY, Wu TS. Melatonin decreases matrix metalloproteinase-9 activation and expression and attenuates reperfusion-induced hemorrhage following transient focal cerebral ischemia in rats. $J$ Pineal Res. 2008;45:459-467.

[32] Paul S, Sharma AV, Mahapatra PD, Bhattacharya P, Reiter RJ, Swarnakar S. Role of melatonin in regulating matrix metalloproteinase-9 via tissue inhibitors of metalloproteinase-1 during protection against endometriosis. J Pineal Res. 2008;44: 439-449. 